\title{
Comportamiento de la infestación por Rhipicephalus sanguineus en perros de La Habana, Cuba
}

\author{
Behavior of Rhipicephalus sanguineus infestation in dogs from Havana, Cuba \\ María Beatriz Rodríguez Alonso ${ }^{1,3}$, Maylín González Navarrete', \\ Evelyn Leonor Reyes Herrera ${ }^{1}$, Elianny Bravo Salabarría ${ }^{2}$
}

\section{Resumen}

Se estudió el comportamiento de la infestación por Rhipicephalus sanguineus en perros de tres clínicas veterinarias de La Habana, entre noviembre de 2016 y octubre de 2017. Se recolectaron 10533 garrapatas en 175 de 300 perros que se examinaron sin discriminar raza, edad o sexo. En el periodo húmedo hubo mayor abundancia de infestación y mayor cantidad de garrapatas $(p<0.001)$, mayor extensidad de infestación $(p<0.08)$, $\mathrm{y}$ alta intensidad de infestación $(\mathrm{p}<0.005)$. La mayor parte de los perros infestados fueron jóvenes, machos y de raza indefinida. Los factores que influyeron en el comportamiento de la infestación por $R$. sanguineus en perros fueron la crianza y el manejo.

Palabras clave: Rhipicephalus sanguineus, infestación, comportamiento, La Habana

\section{AbSTRACT}

The behavior of the infestation by Rhipicephalus sanguineus in dogs from three veterinary clinics in Havana was studied between November 2016 and October 2017. In total, 10533 ticks were collected in 175 of 300 dogs that were examined without

\footnotetext{
${ }^{1}$ Facultad de Medicina Veterinaria, Universidad Agraria de La Habana (UNAH), Mayabeque, Cuba ${ }^{2}$ Departamento de Patología y Cirugía Experimental, Instituto Nacional de Oncología y Radiobiología (INOR), La Habana, Cuba

${ }^{3}$ E-mail: mariabeatriz.ra@gmail.com
}

Recibido: 27 de abril de 2020

Aceptado para publicación: 5 de marzo de 2021

Publicado: 27 de octubre de 2021

CLos autores. Este artículo es publicado por la Rev Inv Vet Perú de la Facultad de Medicina Veterinaria, Universidad Nacional Mayor de San Marcos. Este es un artículo de acceso abierto, distribuido bajo los términos de la licencia Creative Commons Atribución 4.0 Internacional (CC BY 4.0) [https:// creativecommons.org/licenses/by/4.0/deed.es] que permite el uso, distribución y reproducción en cualquier medio, siempre que la obra original sea debidamente citada de su fuente original 
discriminating breed, age or sex. In the wet season there was a greater abundance of infestation and a greater number of ticks $(p<0.001)$, greater extent of infestation $(p<0.08)$, and high intensity of infestation $(p<0.005)$. Most of the infested dogs were young, male and of indefined breed. The factors that influenced the behavior of the infestation by $R$. sanguineus in dogs were breeding and handling.

Key words: Rhipicephalus sanguineus, infestation, behavior, Havana

\section{INTRODUCCIÓN}

Se han descrito infestaciones de la garrapata Rhipicephalus sanguineus (Latreille, 1806) en roedores (Szabó et al., 1995), gatos, conejos (Labruna, 2004), aves (Szabó et al., 2008) y zorros rojos (Ebani et al., 2017). Se le conoce como la garrapata marrón del perro y, precisamente por la asociación cercana entre perros y humanos, $R$. sanguineus está adaptada a entornos urbanos, al ser la especie más cosmopolita (Walker et al., 2005; Rubio et al., 2015).

Así como otras especies de garrapatas, $R$. sanguineus encuentra albergue en Cuba debido a que la isla reúne condiciones favorables por su situación geográfica. Esta especie de garrapata se encuentra distribuida hacia la zona occidental de la isla, y se le halla en las provincias de La Habana, Pinar del Río y el municipio especial Isla de la Juventud (Barros-Batesti et al., 2009). Hallazgos de $R$. sanguineus en perros en la isla han sido reportados por León et al. (2008), Méndez (2011) y Alemán et al. (2014).

A pesar de la gran atención científica que tienen las garrapatas, se han hecho pocos estudios que determinen el comportamiento de la infestación por $R$. sanguineus, específicamente en el área del Caribe (Morales y Cruz, 1998; Klober, 2001; Ramírez et al., 2008; Silveira et al., 2009; Gondard et al., 2017), pero no se dispone de suficientes trabajos que aborden este fenómeno en Cuba (González et al., 2016). Investigaciones en el área, relacionadas con el comportamiento de la infestación por $R$. sanguineus, clasifican las infestaciones como baja, moderada y alta, sin explicar los métodos del análisis, lo que hace imposible utilizar su modelo por no mostrar su metodología, mismo problema que encontraron Huerto-Medina y Dámaso-Mata (2015). El estudio más cercano a estas características fue realizado por Ferrell et al. (2017), quienes utilizan fórmulas estadísticas como abundancia, prevalencia y carga.

Existen diversos criterios sobre cómo influyen las características inherentes al perro y los aspectos de su manejo con la infestación por garrapatas $R$. sanguineus, pero ninguno aplicado al contexto cubano. Por esto, se puede presumir que no se dispone de información científica sobre el comportamiento de la infestación por $R$. sanguineus en perros de La Habana, de allí que el objetivo del presente estudio fue determinar el comportamiento de la infestación por $R$. sanguineus en perros de La Habana, a través de los indicadores epidemiológicos de abundancia, extensidad e intensidad de infestación de la garrapata en perros en los dos periodos del año, así como evaluar la severidad de la infestación y determinar el comportamiento de la infestación según características inherentes al perro y aspectos de su manejo. 


\section{Materiales y Métodos}

Se realizó un estudio descriptivo, transversal y prospectivo en la Facultad de Medicina Veterinaria de la Universidad Agraria de La Habana «Fructuoso Rodríguez Pérez», desde noviembre de 2016 hasta octubre de 2017 (12 meses). Sobre la base de la facilidad del acceso y la posibilidad de contactarse con criadores y veterinarios, se seleccionaron tres clínicas veterinarias ubicadas en los municipios habaneros Playa, Centro Habana y Habana del Este. Se hizo una visita mensual con ocho horas de permanencia, lográndose examinar 300 perros sin discriminar raza, edad o sexo, y que fueron llevados a recibir algún servicio. El muestreo se realizó en presencia de un veterinario y con el acuerdo de los propietarios, quienes fueron encuestados.

Los perros fueron revisados físicamente para detectar la presencia de garrapatas en cualquier estadio de desarrollo. Se recolectó la mayor cantidad posible de las garrapatas utilizando pinzas finas con borde liso ( $\sin$ dientes), introduciendo la pinza entre la cabeza y la piel tal y como lo describe AVEPA (2012). Las garrapatas se colocaron en alcohol a $90^{\circ}$ y se llevaron al Laboratorio de Parasitología de la Unidad Docente «Nazareno», donde fueron clasificadas de acuerdo con el sexo y estadio, identificándose el género y especie a través de las claves taxonómicas de Pérez-Vigueras (1953), Demedio et al. (1984) y Barros-Batesti et al. (2009).

Los datos del estudio fueron divididos en dos periodos: seco (noviembre-abril) y húmedo (mayo-octubre) según las definiciones de Rojas et al. (2011). En el estudio de Ferrell et al. (2017) en Panamá, se determinó la prevalencia, abundancia y carga utilizando las siguientes fórmulas: Abundancia (total de parásitos/total de perros), Prevalencia (número de perros infestados/total de perros) y Carga o intensidad de infestación (total de parásitos/perros infestados). La seve- ridad de la infestación en el perro se evaluó teniendo en cuenta los rangos establecidos por Tinoco et al. (2009) en México, considerando la infestación baja (1-10), media (1130) y alta (más de 30 garrapatas).

Se aplicó una encuesta según lo recomendado por Casas-Anguita et al. (2003). El cuestionario semiestructurado fue elaborado y evaluado previamente con el objetivo de perfeccionarlo en cuanto a la complejidad de las preguntas, secuencia lógica de acuerdo con los objetivos, máxima claridad y adición de eventuales preguntas que pudieran servir como auxilio en la obtención de la información. Los aspectos contenidos en el cuerpo de la escuesta fueron los factores inherentes al perro, tales como sexo, raza y edad; y los relacionados a la crianza y el manejo del perro: hábitat del animal, contacto con otros animales, forma en que los dueños mantienen al perro, salidas a la calle y frecuencia, y productos garrapaticidas utilizados por los dueños.

Los grupos etarios fueron conformados de acuerdo a lo planteado por Pino (2006), quien considera cuatro grupos: cachorros $(<1$ año), jóvenes ( $>1-\leq 5$ años), adultos ( $>5-\leq 10$ años) y gerontes ( $>10$ años). Para la raza se utilizaron las claves publicadas por la Federación Cinológica Internacional (FCI, 2018), donde cualquier perro con raza definida mediante estas claves se agrupa con el acrónimo $\mathrm{CRD}$, aunque sea una raza no reconocida por la federación, y los que no tienen una raza definida con el acrónimo SRD.

Los datos se tabularon y procesaron con los programas de Microsoft Office Access y Excel 2016. Se realizó el análisis estadístico de comparación de proporciones utilizando el programa CompaProWin 2.0.1 y cuando se compararon dos variables se utilizó la prueba de hipótesis con el paquete estadístico Statgraphics Centurion XVI (16.1.11). Para efectos del estudio se consideró un nivel de confianza de $95 \%$. Los resultados se representaron en tablas para su mejor análisis y comprensión. 


\section{Resultados y Discusión}

\section{Especie de Garrapatas}

De las 10533 garrapatas extraídas de los perros se encontró a $R$. sanguineus como única especie de garrapata. En México, Tinoco et al. (2009) observaron que el 100\% de las garrapatas colectadas en 384 caninos callejeros fueron identificadas como $R$. sanguineus. Asimismo, reportes de Brasil muestra que $R$. sanguineus fue la única especie encontrada en perros infestados (Pessoa et al., 2006; Braz et al., 2007; Silveira et al., 2009). Similares hallagos han sido reportados en Venezuela (Ramírez et al., 2008) y Cuba (León et al., 2008; González et al., 2016; Silva et al., 2016). No obstante, Gervasoni et al. (2003) reportaron un caso de infestación cruzada con Amblyomma tigrinum.

\section{Estadios}

La frecuencia de estadios de $R$. sanguineus en los perros infestados según el periodo del año se muestra en el Cuadro 1 . Se observó diferencias altamente significativas $(p=0.00)$ entre todos los estadios de cada uno de los periodos, a diferencia de Braz et al.

Cuadro 1. Frecuencia de garrapatas Rhipicephalus sanguineus, según estadio de desarrollo y periodo del año, en perros atentidos en clínicas veteriarias de $\mathrm{La}$ Habana, Cuba (noviembre 2016 - octubre 2017)

\begin{tabular}{lcc}
\hline & $\begin{array}{c}\text { Periodo } \\
\text { seco }\end{array}$ & $\begin{array}{c}\text { Periodo } \\
\text { húmedo }\end{array}$ \\
\hline Ninfa & $0.22^{\mathrm{a}}$ & $0^{\mathrm{b}}$ \\
Larva & $1.44^{\mathrm{a}}$ & $0.38^{\mathrm{b}}$ \\
Imago macho & $37.98^{\mathrm{a}}$ & $43.37^{\mathrm{b}}$ \\
Imago hembra & $60.36^{\mathrm{a}}$ & $56.25^{\mathrm{b}}$ \\
\hline a,b Promedios entre filas con diferente letra son \\
\multicolumn{2}{l}{ estadísticamente diferentes ( $\mathrm{p}<0.01)$}
\end{tabular}

(2007) quenes no encontraron diferencias entre estadios por efecto de las temporadas seca y húmeda.

Pino (2006) y Ramírez et al. (2008) encontraron una mayor cantidad de $R$. sanguineus hembras que machos, mientras que Ferrell et al. (2017) en Panamá no encontraron diferencias entre sexos y Lorusso et al. (2010) en Italia reportaron una mayor frecuencia de machos. De otra parte, García et al. (2007) reportaron una mayor frecuencia de estadios inmaduros, a diferencia de los hallazgos de Silveira et al. (2009), Lorusso et al. (2010) y Ferrell et al. (2017) quienes encontraron una mayor frecuencia de estadios adultos que de ninfas y larvas en perros parasitados.

\section{Indicadores}

En el Cuadro 2 se muestran los resultados del cálculo de la abundancia, extensidad e intensidad de infestación por $R$. sanguineus en perros durante los periodos seco y húmedo. La cantidad de garrapatas del periodo húmedo fue significativamente mayor $(p<0.05)$ que durante el periodo seco. Sin embargo, no hubo diferencia significativa entre las proporciones de los perros infestados y la extensidad entre periodos.

Estos resultados concuerdan con lo planteado por Rojas (2001) y Rubio et al. (2015), quienes explican que, aunque $R$. sanguineus puede alimentarse durante todo el año en climas meridionales (zonas tropicales y subtropicales), su presencia tiene mayor prevalencia durante la primavera y el verano. Asimismo, Cruz-Vázquez y GarcíaVázquez (1999) reportaron un menor número de garrapatas $R$. sanguineus en perros en México durante el invierno en comparación con el resto del año. No obstante, Braz et al. (2007) en su estudio en perros de Goiás, Brasil, no encontraron diferencias significativas en el número de garrapatas entre el periodo húmedo y caluroso con el periodo seco y frio. 
Cuadro 2. Comportamiento de los indicadores epidemiológicos: abundancia, extensidad e intensidad de infestación por Riphicephalus sanguineus en perros de La Habana, en los periodos seco y húmedo

\begin{tabular}{lcccccc}
\hline Periodo & $\begin{array}{c}\text { Garrapatas } \\
(\mathrm{n})\end{array}$ & $\begin{array}{c}\text { Perros } \\
\text { muestreados }\end{array}$ & $\begin{array}{c}\text { Perros } \\
\text { infestados }\end{array}$ & Abundancia & $\begin{array}{c}\text { Extensidad } \\
(\%)\end{array}$ & Intensidad \\
\hline Seco & $4,937^{\mathrm{a}}$ & 150 & 80 & $32.91^{\mathrm{a}}$ & 53.3 & 61.71 \\
Húmedo & $5,596^{\mathrm{b}}$ & 150 & 95 & $37.31^{\mathrm{b}}$ & 63.3 & 58.91 \\
\hline Total & 10,533 & 300 & 175 & 35.11 & 58.3 & 60.19 \\
\hline
\end{tabular}

a,b Promedios entre columnas con diferente letra son estadísficamente diferentes $(p<0.05)$

\section{Severidad de Infestación}

De 175 perros infestados el día del muestreo, el $59.4 \%$ tenía 30 garrapatas o más, de los cuales $13.7 \%$ estaba infestado con 100 200 garrapatas, $3.4 \%$ con $200-300$ y $0.6 \%$ con más de 300 garrapatas. Los perros con más de 100 garrapatas representaron el 17.7\% del total de perros infestados, indicando la gravedad de algunas infestaciones. Incluso, en un caso, se recuperaron 517 garrapatas de un perro. Klober (2001) reportó valores que van entre 49 y 210 garrapatas por perro, considerándolos como un grado de infestación alto, mientras que Ramírez et al. (2008) encontraron un grado de infestación bajo (255 garrapatas por perro).

El Cuadro 3 muestra la cantidad de perros infestados según la severidad de infestación, observándose diferencias significativas en el valor alto de cada uno de los periodos, especialmente en la época húmeda. Por otro lado, en el total de casos del año, la mayor frecuencia de casos fue con severidad alta en comparación con los niveles de severidad baja y media $(\mathrm{p}<0.05)$. Tinoco et al. (2009) reportaron una frecuencia de 5.36\% de casos con severidad alta, $16.07 \%$ de severidad media y $78.57 \%$ de everidad baja, valores muy diferentes a los del presente estudio.

\section{Factores Inherentes al Perro}

\section{Grupos etarios}

El Cuadro 4 muestra una mayor frecuencia de perros infestados por $R$. sanguineus en el grupo etario de jóvenes con relación a los otros grupos $(\mathrm{p}<0.05)$. Asimismo, el grupo etario cachorros y gerontes mostraron la menor frecuencia de infestación sin diferencias significativas entre ellos. Al respecto, Huerto-Medina y Dámaso-Mata (2015) plantean que los perros de mediana edad tienen mayores oportunidades de estar expuestos a $R$. sanguineus que los cachorros, por la costumbre de los dueños de sacar a la calle a los animales cuando completan sus vacunas por considerar que tienen menos riesgo de contraer enfermedades infecciosas.

Sexo y raza

El 52.3\% de los 330 perros muestreados fue macho; asimismo, el 44.0 fue SRD y $56.0 \%$ CRD. Dentro de este grupo encontramos a American Pitbull Terrier, American Staffordshire Terrier, Basset Hound, Bichón Habanero, Boxer, Bulldog Francés, Chihuahua, Perro sin pelo del Perú, Chow Chow, Cocker Spaniel, Dachshund, Dálmata, Doberman Pinscher, Golden Retriever, Gran 
Cuadro 3. Niveles de severidad de infestación por garrapatas Riphicephalus sanguineus en 175 perros de La Habana, Cuba

\begin{tabular}{llcc}
\hline Periodo & Nivel & $\begin{array}{c}\text { Perros } \\
\text { infestados }\end{array}$ & Proporción \\
\hline Seco & Bajo & 24 & $0.30^{\mathrm{c}}$ \\
& Medio & 16 & $0.20^{\mathrm{c}}$ \\
& Alto & 40 & $0.50^{\mathrm{b}}$ \\
Húmedo & Bajo & 5 & $0.05^{\mathrm{d}}$ \\
& Medio & 26 & $0.27^{\mathrm{c}}$ \\
& Alto & 64 & $0.67^{\mathrm{a}}$ \\
\hline Total & Bajo & 29 & $0.17^{\mathrm{b}}$ \\
& Medio & 42 & $0.24^{\mathrm{b}}$ \\
& Alto & 104 & $0.59^{\mathrm{a}}$ \\
\hline a,b,c,d Promedios con & diferente letra son \\
estadísficamente diferentes $(\mathrm{p}<0.05)$ &
\end{tabular}

Danés, Labrador Retriever, Perro de Presa Canario, Pastor Alemán, Pastor Belga Malinois, Pequinés, Pointer Inglés, Poodle, Rottweiler, Schnauzer estándar, Shar Pei, Shih Tzu, Husky Siberiano, Tibetan Spaniel, Toy Fox Terrier y Yorkshire Terrier. En este sentido, Pino (2006) en su estudio, encontró que el $64 \%$ de la población muestreada se clasificó como raza pura y el $36 \%$ como perro crio1lo. Dentro de los perros CRD infestados, el Pequinés fue la raza más frecuentemente infestada (40.5\%), seguido por el Pastor Alemán $(11.4 \%)$.

El 58.3\% de los perros estaba infestado por $R$. sanguineus $(\mathrm{n}=175), \mathrm{y}$ de estos, el $43.4 \%$ fue hembra y el $56.6 \%$ macho. Así mismo, $45.1 \%$ fue CRD y 54.9 SRD. Si bien no hubo diferencia significativa entre la proporción de perros muestreados por sexo $(\mathrm{p}<0.126)$, la proporción de perros machos infestados fue significativamente mayor $(p<0.05)$. En el caso de los grupos raciales, si bien el mayor número de perros muestreados fue CRD (56\%), el 54.9\% de los perros parasitados correspondió al grupo SRD $(\mathrm{p}<0.05)$

Silveira et al. (2009) reportaron en Brasil, al ingual que en el presente estudio, mayor frecuencia de perros machos infestados por $R$. sanguineus $(58.3 \%)$ en comparación con perras hembras infestadas, mientras que en Venezuela, Ramírez et al. (2008) no encontraron diferencias significativas entre perros infestados con garrapatas por efecto del sexo del animal. Con relación a los grupos raciales, Silveira et al. (2009) y Pessoa et al. (2006), reportaron una mayor frecuencia de animales SRD infestados (66 y $65 \%$, respectivamente) que CRD, en tanto que Ramírez et al. (2008) no encontraron diferencias significativas entre infestaciones en perros de razas puras $(48.4 \%)$ y perros mestizos $(51.6 \%)$.

\section{La Crianza y el Manejo del Perro}

\section{Hábitat del animal}

El 24.7\% de los perros muestreados viven en patio cementado, $23.3 \%$ en patio de tierra, $1.1 \%$ en casa de madera, $22.7 \%$ en casa de mampostería, $21.4 \%$ en apartamento, $3.6 \%$ en techo/azotea, $3.3 \%$ en otros (cuarto, finca y construcción). De todos estos, $17.8 \%$ vive en combinaciones con más de un hábitat. La relación entre infestación con $R$. sanguineus y el tipo de hábitat de los perros se muestra en el Cuadro 5.

Rezende (2012), en su estudio en Rio de Janeiro, Brasil, determina que el $60.4 \%$ $(188 / 311)$ de los perros habitaban en locales con presencia de tierra y cementados, siendo esta variedad altamente asociada a las condiciones ecológicas del ambiente para el ciclo del vector, aliada a las dificultades para tratamiento garrapaticida en el ambiente. 
Cuadro 4. Infestación por garrapatas Riphicephalus sanguineus en 300 perros de La Habana, Cuba según el grupo etario

\begin{tabular}{lccc}
\hline & Muestrados (n) & Infestados $(\mathrm{n})$ & Proporción \\
\hline Cachorros & 45 & 23 & $0.51^{\mathrm{b}}$ \\
Jóvenes & 130 & 120 & $0.92^{\mathrm{a}}$ \\
Adultos & 110 & 23 & $0.20^{\mathrm{c}}$ \\
Gerontes & 15 & 9 & $0.6^{\mathrm{b}}$ \\
\hline
\end{tabular}

a,b,c Promedios con diferente letra son estadísficamente diferentes $(p<0.05)$

Cuadro 5. Frecuencia de perros infestados por garrapatas Riphicephalus sanguineus según el tipo de hábitat (Habana, Cuba)

\begin{tabular}{lccc}
\hline & Muestrados (n) & Infestados $(\mathrm{n})$ & Proporción \\
\hline Patio de tierra & 70 & 45 & $0.64^{\mathrm{b}}$ \\
Patio cementado & 74 & 41 & $0.55^{\mathrm{b}}$ \\
Casa de mampostería & 68 & 40 & $0.59^{\mathrm{b}}$ \\
Apartamento & 64 & 33 & $0.52^{\mathrm{b}}$ \\
Otros & 10 & 8 & $0.80^{\mathrm{ab}}$ \\
Techo/azotea & 11 & 5 & $0.45^{\mathrm{ab}}$ \\
Casa de madera & 3 & 3 & $1^{\mathrm{a}}$ \\
\hline
\end{tabular}

a,b Proporciones con diferentes letras son estadísticamente diferentes $(p<0.05)$

Cuadro 6. Frecuencia de perros infestados por garrapatas Riphicephalus sanguineus que tienen contacto con otros animales dentro de la casa (Habana, Cuba)

\begin{tabular}{lccc}
\hline & Muestrados (n) & Infestados (n) & Proporción \\
\hline 1 perro & 104 & 59 & $0.57^{\mathrm{a}}$ \\
2 perros & 7 & 5 & $0.71^{\mathrm{a}}$ \\
3 perros & 4 & 3 & $0.75^{\mathrm{a}}$ \\
Perros callejeros & 12 & 8 & $0.67^{\mathrm{a}}$ \\
1 gato & 22 & 15 & $0.68^{\mathrm{a}}$ \\
2 gatos & 3 & 2 & $0.67^{\mathrm{a}}$ \\
Gerbillos & 2 & 1 & $0.5^{\mathrm{a}}$ \\
Jicotea & 2 & 1 & $0.5^{\mathrm{a}}$ \\
Pollo & 1 & 1 & $1^{\mathrm{a}}$ \\
Vaca & 1 & 1 & $1^{\mathrm{a}}$ \\
\hline
\end{tabular}

a,b Proporciones con diferentes letras son estadísticamente diferentes $(p<0.05)$ 
Contacto con otros animales dentro de la casa

El $46.3 \%$ de los perros muestreados y el $54.9 \%(96 / 175)$ de los perros infestados tienen contacto con otros animales dentro de la vivienda, mayormente con otros perros propios de la casa o callejeros, así como con gatos (Cuadro 6).

Rezende (2012) plantea que el contacto con otros animales es una variable importante para el mantenimiento de la infestación, principalmente cuando el manejo garrapaticida ambiental y animal son deficientes, $o$ es realizado solo en alguno de los animales que viven en el mismo ambiente.

\section{Manejo del perro}

El 72.7\% de los propietarios de los perros los mantienen sueltos, $11.3 \%$ amarrado y $16.0 \%$ los tiene amarrado parte del tiempo. El Cuadro 7 muestra la comparación de las proporciones entre la forma en que mantienen a los perros infestados por $R$. sanguineus con respecto al total de infestados $(\mathrm{p}<0.05)$.

\section{Salidas y frecuencia}

De los 300 perros muestreados, el $70.8 \%$ sale, siendo una vez al día el $41.7 \%$, dos veces al día el $29.2 \%$ y $29.2 \%$ sin restricción. En el caso de los perros infestados, el $41.1 \%$ nunca sale y del $58.9 \%$ que sale,
$62.1 \%$ de estos sale acompañado y $37.8 \%$ sin restricción. Se deduce, por lo tanto, que el mayor porcentaje de perros infestados tiene acceso a la calle y contacto con otros animales directa o indirectamente.

Sobre este tema, Huerto-Medina y Dámaso-Mata (2015) explican que la condición del estilo de vida «callejero» del perro es un factor de riesgo, debido a que cuando un animal se encuentra fuera de su hogar, al estar en contacto con perros «vagabundos» que por lo general siempre están infestados de garrapatas.

\section{Productos garrapaticidas utilizados}

En las regiones tropicales y subtropicales se ha dificultado el control de $R$. sanguineus debido a que su alta tasa de reproducción, infestación y longevidad le ha permitido adaptar su ciclo biológico al ambiente doméstico y peridoméstico, en donde las condiciones ecológicas le son favorables y le permiten el estrecho contacto con perros y humanos (Izquierdo, 2012). Se conoce que una garrapata adulta puede sobrevivir entre 155 y 568 días sin alimentarse, lo que dificulta el control, principalmente en los estadios de vida libre (Dolz et al., 2013). Cuba es un país tropical y el control de $R$. sanguineos en perros se basa teniendo en cuenta las recomendaciones internacionales según el ciclo biológico de la especie para áreas climáticas similares (Roque-López, 2019).

Cuadro 7. Infestación por garrapatas Riphicephalus sanguineus en 300 perros de La Habana, Cuba, según el tipo de manejo del perro

\begin{tabular}{lccc}
\hline & Muestrados (n) & Infestados (n) & Proporción \\
\hline Suelto & 218 & 138 & $0.63^{\mathrm{a}}$ \\
Amarrado & 34 & 16 & $0.47^{\mathrm{ab}}$ \\
Amarrado y suelto & 48 & 21 & $0.44^{\mathrm{b}}$ \\
\hline
\end{tabular}

a,b,c Promedios con diferente letra son estadísficamente diferentes $(p<0.05)$ 
Cuadro 8. Frecuencia de perros infestados por garrapatas Riphicephalus sanguineus con relación a salidas a la calle (La Habana, Cuba)

\begin{tabular}{lccc}
\hline & Muestrados (n) & Infestados $(\mathrm{n})$ & Proporción \\
\hline Salen & 212 & 103 & $0.49^{\mathrm{bc}}$ \\
No salen & 88 & 72 & $0.82^{\mathrm{a}}$ \\
Acompañado & 151 & 64 & $0.42^{\mathrm{c}}$ \\
Sin restricción & 62 & 39 & $0.63^{\mathrm{b}}$ \\
1 vez al día & 88 & 40 & $0.45 \mathrm{~b}^{\mathrm{c}}$ \\
2 veces al día & 62 & 24 & $0.39^{\mathrm{c}}$ \\
\hline
\end{tabular}

$a, b, c$ Promedios con diferente letra son estadísficamente diferentes $(p<0.05)$

Lord (2014), en su investigación llevada a cabo en la Universidad de Florida, trató aleatoriamente a perros con una mezcla «tropical» de fipronil y permetrina (6.7 a 13.4 $\mathrm{mg} / \mathrm{kg}$ y 60 a $120 \mathrm{mg} / \mathrm{kg}$, respectivamente) con una eficacia mayor del $90 \%$ a las 24 horas de la aplicación. En la presente investigación, al $47.3 \%$ de los perros se les aplica fipronil, al 34.0\% Amitrazy al restante 18.7\% otros métodos de control. Ciertamente en algunos casos se utilizaba más de un producto garrapaticida en los perros. Además, se resalta la utilización de productos y métodos no convencionales como el alcohol, agua de abate, petróleo, y tintura de pino macho con agua y extracción manual para el control de las garrapatas.

Entre los que utilizan fipronil, aproximadamente el 30\% lo aplica semanalmente, 54\% mensualmente y $18 \%$ de manera trimestral. En el caso del Amitraz, el 33\% lo aplica 2 veces por semana, $57 \%$ cada 7 días, $4 \%$ cada 3 meses y $6 \%$ lo utilizó solo una vez en la vida del perro. El desconocimiento de los dueños sobre la utilización de productos para el control de las garrapatas se observó en la disparidad o variedad de frecuencias de reactivación de los productos garrapaticidas, así como en los nombres coloquiales que utilizan para referirse a estas sustancias.
La pluralidad de métodos de control es un fenómeno encontrado también por Pino (2006), quien agrega que entre estas diversas medidas se encuentran jabones ixodicidas (25\%), baños garrapaticidas (23\%), uso combinado de jabón insecticida, baño garrapaticida, ivermectina inyectable al $1 \%$ y pipeta Front Line (16\%), jabones ixodicidas en conjunto con ivermectina inyectable $(13 \%)$, collar garrapaticida junto con jabón ixodicida (13\%), y finalmente, aplicación subcutánea de ivermectina (7\%); sin embargo, no se han encontrado reportes sobre la utilización de productos alternativos tan peculiares como el petróleo o agua de abate.

\section{Conclusiones}

- El indicador epidemiológico abundancia de infestación por Rhipicephalus sanguineus fue significativamente superior en el periodo húmedo con relación al seco.

- La severidad de infestación por $R$. sanguineus fue alta en ambos periodos con un porcentaje significativamente mayor en el periodo húmedo.

- La crianza y el tipo de manejo del perro fueron factores que influyeron en el comportamiento de la infestación por $R$. sanguineus. 


\section{Literatura Citada}

1. Alemán Y, Martínez S, Corona B. 2014. Las garrapatas de interés veterinario en Cuba, y su importancia en las condiciones climáticas cambiantes. REDVET 15(2). [Internet]. Disponible en: http://www.veterinaria.org/revistas/ redvet/n020214.html

2. [AVEPA] Asociación de Veterinarios Españoles Especialistas en Pequeños Animales. 2012. Actualización en diagnóstico y control de enfermedades infecciosas en el perro y gato. AVEPA. 48 p.

3. Barros-Batesti D, Reyes M, Famadas K, Onofrio V, Beati L, Guglielmone A. 2009. The ixodid ticks (Acari: Ixodidae) of Cuba. Syst Appl Acarol-UK 14: 101128. doi: 10.11158/saa.14.2.3

4. Braz C, Nogueira I, Ferreira de Oliveira V, Coelho Linhares $G$, Borges de Menezes L, Ferreira Borges $L$. 2007. Seasonal dynamics of Rhipicephalus sanguineus (Acari: Ixodidae) in dogs from a police unit in Goiânia, Goiás, Brazil. Cienc Rural 37: 464-469.

5. Casas-Anguita J, Repullo-Labrador J, Donado-Campos J. 2003. La encuesta como técnica de investigación. Elaboración de cuestionarios y tratamiento estadísticos de los datos. Aten Prim 31: 527-538. doi: 10.1016/S0212-6567(03)70728-8

6. Cruz-Vazquez, C, Garcia-Vazquez, Z. 1999. Seasonal distribution of Rhipicephalus sanguineus ticks (Acari: Ixodidae) on dogs in an urban area of Morelos, Mexico. Exp Appl Acarol 23: 277-280. doi: 10.1023/a:1006075232455

7. Demedio J, Meireles T, Roque L. 1984. Manual de prácticas de parasitología I. Cuba: Univ. Agraria de La Habana

8. Dolz G, Ábrego L, Romero LE, Campos-Calderón L, Bouza-Mora L, Jiménez-Rocha AE. 2013. Ehrlichiosis y anaplasmosis en Costa Rica. En: IV Congreso Latinoamericano de Enfermedades Rickettsiales. San José, Costa Rica.
9. Ebani VV, Rocchigiani G, Nardoni S, Bertelloni F, Vasta V, Papini RA, Verin R, et al. 2017. Molecular detection of tick-borne pathogens in wild red foxes (Vulpes vulpes) from Central Italy. Acta Trop 172: 197-200. doi: 10.1016/ j.actatropica.2017.05.014

10. Estrada P. 2011. Introducción a las garrapatas: consideraciones generales. Argos 118: 56-58.

11. [FCI] Fédération Cynologique Internationale. 2018. Listado de razas Federación Cinológica Internacional (FCI). Internet]. Disponible en: http:// www.fci.be/es/

12. Ferrell AM, Brinkerhoff RJ, Bernal J, Bermúdez SE. 2017. Ticks and tickborne pathogens of dogs along an elevational and land-use gradient in Chiriquí province, Panamá. Exp Appl Acarol 71: 371-385. doi: 10.1007/s10493017-0116-Z

13. García M, Moissant E, Pérez A, Quijada J, Simoes D, García H. 2007. Comportamiento natural de las fases no parasísticas de Rhipicephalus sanguineus (Latreille, 1806) (Acari: Ixodidae) en un bioterio canino de Venezuela. Rev Cient-Fac Cien V 17: 566-571.

14. Gervasoni $S$, Guglielmone $A A$, Tarabla HD, Ruiz MF. 2003. Factors associated with Rhipicephalus sanguineus (Latreille, 1806) household infestation. In: Proc 10th International Symposium on Veterinary Epidemiology and Economics. Viña del Mar, Chile.

15. Gondard M, Cabezas-Cruz A, Charles RA, Vayssier-Taussat M, Albina E, Moutailler S. 2017. Ticks and tick-borne pathogens of the Caribbean: current understanding and future directions for more comprehensive surveillance. Front Cell Infect Microbiol 7: 490. doi: 10.3389/ fcimb.2017.00490

16. González N, Díaz C, Bezerra D, Sandes P, Luis M, Roque L, Henrique F. 2016. Molecular detection of Ehrlic-hia canis and Babesia canis vogeli in Rhipicephalus sanguineus sensu lato ticks from Cuba. Rev Bras Med Vet 38: 63-67. 
17. Huerto-Medina E, Dámaso-Mata B. 2015. Factores asociados a la infección por Ehrlichia canis en perros infestados con garrapatas en la ciudad de Huánuco, Perú. Rev Peru Med Exp Salud Publica 32: 756-760.

18. Izquierdo C. 2012. Importancia de la garrapata (Rhipicephalus sanguineus) como vector de enfermedades infecciosas en la clínica de perros y en la salud pública: estudio recapitulativo. Tesis de Grado. Veracruz, México: Univ. Veracruzana.

19. Klober R. 2001. Garrapatas en caninos. Un estudio en Maracay, estado Aragua. Maracay, Venezuela. Tesis de Grado. Univ. Central de Venezuela. 65 p.

20. Labruna M. 2004). Biologica-ecología de Rhipicephalus sanguineus (Acari: Ixodidae). En: XIII Congresso Brasileiro de Parasitologia Veterinária. Brasil.

21. León A, Demedio J, Márquez M, Castillo E, Perera A, Zuaznaba $O$, et al. 2008. Diagnóstico de Ehrlichiosis en caninos en la ciudad de La Habana. REDVET 3(5). [Internet]. Disponible en: http://www.veterinaria.org/revistas/ recvet $/ \mathrm{n} 050508 . \mathrm{html}$

22. Lord CC. 2014. Brown dog tick, Rhipicephalus sanguineus Latreille (Arachnida: Acari: Ixodidae). Featured Creatures. [Internet]. Disponible en: https://entnemdept.ufl.edu/creatures/ urban/medical/brown_dog_tick.htm Obtenido de http://entomology.ifas.uû.edu/ creatures

23. Lorusso V, Dantas-Torres F, Lia RP, Tarallo VD, Mencke N, Capelli G, Otranto D. 2010. Seasonal dynamics of the brown dog tick, Rhipicephalus sanguineus, on a confined dog population in Italy. Med Vet Entomol 24: 309315. doi: 10.1111/j.1365-2915.2010.00885.x

24. Méndez M. 2011. Control de Ixodidos y enfermedades asociadas. En: VII Congreso Internacional de Ciencias Veterinarias. La Habana, Cuba.
25. Morales M, Cruz C. 1998. Fluctuaciones poblacionales de Rhipicephalus sanguineus, garrapata parásita de perros, en el valle de Cuernavaca, Morelos, México. Estudio preliminar. Vet Méx 29: 299-301.

26. Pérez-Vigueras. 1953. Los ixodidos y culicidos de Cuba, su historia natural y médica. En: Pérez V (ed). Rhipicephalus sanguineus. La Habana, Cuba. p 94-98.

27. Pessoa FS, Robin MH, Mayumi MI, Oliveira CS, Montanari JR, Rigo L, Aguena F. 2006. Identificação de hemoparasitos e carrapatos de caes procedentes do Centro de Controle de Zoonoses de Campo Grande Estado do Mato Grosso do Sul, Brasil. These de Mestrado. Brasil: Univ. Federal de Mato Grosso do Sul. 54 p.

28. Pino L. 2006. Principales géneros de garrapatas identificados en perros de árrea urbana atendidos en consulta privada y su asociación con infecciones hemoparasitarias. Tesis de Grado. Veracruz: Univ. Veracruzana.

29. Ramírez R, Chacín E, Barboza G, Fernández, G, Valera Z, Villalobos A, Angulo F. 2008. Garrapatas (Acari: Ixodidae) recolectadas en caninos bajo asistencia veterinaria en Maracaibo, Venezuela. Rev Cient-Fac Cien V 18: 267-270

30. Rezende J. 2012. Aspectos epidemiológicos da Babesia canis vogeli em cães da baixada fluminense, RJ. Tese de Doutor em Ciências. Brasil: Universidade Federal Rural do Rio de Janeiro. 121 p.

31. Rojas E. 2001. Info Merial: Las garrapatas II parte. [Internet]. Disponible en: http://www.webveterinaria.com/merial/ GarrapataII.pdf

32. Rojas N, Hernández Rodríguez G, Martínez Mojena A, López Anzardo R, Morejón M, Pazos Rodríguez, R. 2011. Fitotecnia de los pastos y forrajes. La Habana, Cuba: Pueblo y Educación. 488 p. 
33. Roque-López E. 2019. Las garrapatas en los animales domésticos. La Habana, Cuba: Univ. de Agraria de La Habana. 60 p.

34. Rubio MC, Gaxiola SM, Enríquez I, Cota SC, Castro del Campo N. 2015. Rhipicephalus sanguineus en caninos en Sinaloa, México. REDVET 16(3). [Internet]. Disponible en: https:// www.redalyc.org/pdf/636/63638740003.pdf

35. Silva CBD, Santos HA, Navarrete $M G$, Ribeiro CCDU, Gonzalez BC, Zaldivar MF, Pires MS, et al. 2016. Molecular detection and characterization of Anaplasma platys in dogs and ticks in Cuba. Ticks Tick Borne Dis 7: 938944. doi: 10.1016/j.ttbdis.2016.04.012

36. Silveira JA, Passos LM, Ribeiro MF. 2009. Population dynamics of Rhipicephalus sanguineus (Latrielle, 1806) in Belo Horizonte, Minas Gerais state, Brazil. Vet Parasitol 161: 270-275. doi: 10.1016/j.vetpar.2009.01.028

37. Szabó MP, Mukai L, Rosa P. 1995. Differences in the acquired resistance of dogs, hamsters, and guinea pigs to repeated infestations with adult ticks Rhipicephalus sanguineus (Acari: Ixodidae). Braz J Vet Res Anim Sci 32: 43-50. doi: 10.11606/issn.1678-4456.bjvras. 1994.52089

38. Szabó MP, Tolesano-Pascoli GV, Marçal O, Franchin A, Torga K. 2008. Brown dog tick Rhipicephalus sanguineus parasitizing the bird Coereba flaveola in the Brazilian cerrado. Cienc Rural 38: 543-545. doi: 10.1590/ S0103-84782008000200041

39. Tinoco L, Quiroz H, Quintero M, Rentería T, Barreras A, Romano M, et al. 2009. Prevalencia de infestación de garrapatas (Rhipicephalus sanguineus) en perros y su asociación a factores de riesgo en la zona urbana de Mexicali, Baja California, México. En: VIII Congreso Nacional de Parasitología Veterinaria. México.

40. Walker J, Keirans J, Horak I. 2005. The genus Rhipicephalus (Acari: Ixodidae): a guide to the brown ticks of the world. Cambridge: Cambridge University Press. 643 p. 